\title{
Oscillation Criteria for Higher Order Functional Equations
}

\author{
Lin Jingjie ${ }^{1}$, Wu Simin ${ }^{1}, \&$ Lin Quanwen ${ }^{1}$
}

${ }^{1}$ Department of Mathematics, Science of School, Guangdong University of Petrochemical Technology, Maoming of Guangdong China

Correspondence: Lin Quanwen, Guandu Second Road, Department of Mathematics, Science of School, Guangdong University of Petrochemical Technology, Maoming of Guangdong China. E-mail: linquanwen@126.com

Received: February 11, 2019 Accepted: March 4, 2019 Online Published: March 24, 2019

doi:10.5539/jmr.v11n2p135

URL: https://doi.org/10.5539/jmr.v11n2p135

\section{Abstract}

This paper mainly studies oscillatory of all solutions for a class higher order linear functional equations of the form

$$
x(g(t))=P(t) x(t)+\sum_{i=1}^{m} Q_{i}(t) x\left(g^{k+i}(t)\right)
$$

Where $P, Q, g:\left[t_{0}, \infty\right] \rightarrow R^{+}=[0, \infty]$ are given real valued functions and $g(t) \neq t, \lim _{t \rightarrow \infty} g(t)=\infty$.

Some sufficient conditions are obtained. Our results generalize or improve some results in some literature given. An example is also given to illustrate the results.

Keywords: oscillation, high order, linear, functional equations

\section{Introduction}

Consider the high order functional equation :

$$
x(g(t))=P(t) x(t)+\sum_{i=1}^{m} Q_{i}(t) x\left(g^{k+i}(t)\right)
$$

$P, Q_{i}: I \rightarrow(0, \infty)(i=1,2,3, \ldots, m), g: I \rightarrow I$, which is a given function and $x(t)$ is an unknown function. $I$ is an unbounded subset in $(0, \infty) . g(t) \neq t, \lim _{t \rightarrow \infty} g(t)=\infty(t \in I), g^{m}$ that $m$ times iteration of function $g$ means:

$$
g^{0}(t)=t, g^{i+1}(t)=g\left(g^{i}(t)\right), t \in I, i=1,2 \ldots . . m .
$$

As a solution of equation (1.1) if $x: I \rightarrow R$, such that:

$$
\sup \left\{|x(s)|: s \in I_{t_{0}}=\left[t_{0}, \infty\right) \bigcap I\right\}>0 \text { for } \forall t_{0} \in(0, \infty)
$$

is setting up, satisfied (1.1) for $t \in I$., we call this solution is oscillatory.

When $i=1, k=1$ :

$$
x(g(t))=P(t) x(t)+Q(t) x\left(g^{2}(t)\right),
$$

Where $Q: I \rightarrow(0, \infty)$ is a functional equation of a given.

In 1994, Golda and Werbowski firstly did the research of the oscillation of the solutions of equation (1.2), and we could know it from their research. If

$$
\limsup _{t \rightarrow \infty} Q(t) P(g(t))>1
$$

or 


$$
\liminf _{t \rightarrow \infty} Q(t) P(g(t))>\frac{1}{4}
$$

every solution of function (1.2) will oscillate.

At the same time they also will be extended (1.3) to:

$$
\limsup _{t \rightarrow \infty}\left\{(t) P(g(t))+\sum_{i=0}^{k} \prod_{j=0}^{i} Q\left(g^{j+1}(t)\right) P\left(g^{j+2}(t)\right)\right\}>1
$$

There $k \geq 0$ is an integer.

In 1995, Nowakowska and Werbowsk [2] extended the condition (1.4) to

$$
\begin{gathered}
x(g(t))=P(t) x(t)+\sum_{i=1}^{k} Q_{i}(t) x\left(g^{k+1}(t)\right) \\
\liminf _{t \rightarrow \infty} \sum_{i=1}^{k} Q_{i}(t) \prod_{j=1}^{k} P\left(g^{j}(t)\right)>\frac{1}{4}
\end{gathered}
$$

or

$$
\liminf _{t \rightarrow \infty} \sum_{i=0}^{k-1} G\left(g_{i}(t)\right) \prod_{j=1}^{k} P\left(g^{i+j}(t)\right)>\left(\frac{k}{k+1}\right)^{k+1}
$$

where

$$
G(t)=\sum_{n=1}^{k-1} Q_{n}(t) Q_{k-n}\left(g^{n}(t)\right)+Q_{k}(t)
$$

In 1999, Zhou Yong and Yu Yuanhong [3] research the oscillation of solution of equation (1.1). They proved the oscillation equation (1.1). If

$$
\liminf _{t \rightarrow \infty} \sum_{i=1}^{m} Q_{i}(t) \prod_{j=1}^{k+i-1} P\left(g^{j}(t)\right)=A>\frac{k^{k}}{(k+1)^{k+1}}
$$

or

$$
0 \leq A \leq \frac{k^{k}}{(k+1)^{k+1}}, \limsup _{t \rightarrow \infty} \sum_{i=1}^{m} Q_{i}(t) \prod_{j=1}^{k+i-1} P\left(g^{j}(t)\right)>\frac{1}{[\lambda(A)]^{k}}
$$

$\lambda$ is the only real root of $A \lambda^{k+1}-\lambda+1=0$ in $\left[1,((k+1) A)^{-\frac{1}{k}}\right.$.

In recent years, the oscillation of the function equation has become a hot topic for mathematicians (see literature s[4-12]), theyobtain some oscillate criterion of the solutions of various linear advanced functional equations. Inspired by them, we obtaint some new results.

\section{Results and Ploofs}

Consider the high order functional equation (1.1)

$$
x(g(t))=p(t) x(t)+\sum_{i=1}^{m} Q_{i}(t) x\left(g^{k+i}(t)\right)
$$

let

$$
\mu=\liminf _{t \rightarrow \infty} \sum_{i=1}^{m} Q_{i}(t) \prod_{j=1}^{k+i-1} P\left(g^{j}(t)\right)
$$

and

$$
\omega_{1}(t)=\frac{p(t) x(t)}{x(g(t))}, \quad \omega_{2}(t)=\frac{x\left(g^{2}(t)\right)}{x(g(t))} \sum_{i=1}^{m} Q_{i}(t) \prod_{j=2}^{k+i-1} P\left(g^{j}(t)\right)
$$

Lemma 1.1 Assume $0 \leq \mu \leq \frac{k^{k}}{(k+1)^{k+1}}, x(t)$ is the final positive solutions of equation (1.1),then

$$
\limsup _{t \rightarrow \infty} \omega_{i}(t) \leq d, i=1,2
$$


PROOF: By equation (1.1), we know:

$$
x(g(t)) \geq p(t) x(t)
$$

then: $\quad d_{1}=1 \geq \frac{P(t) x(t)}{x(g(t))}, \frac{x(g(t))}{x(t)} \geq P(t) d_{1}^{-1}$, thus (1.1) is established when $\mu=0 ; \quad i=1$

In addition, we prove $0<\mu \leq \frac{k^{k}}{(k+1)^{k+1}}$ for any $\varepsilon \in(0, \mu)$ when $t \rightarrow \infty$. Thus

$$
\sum_{i=1}^{m} Q_{i}(t) \prod_{j=1}^{k+i-1} P\left(g^{j}(t)\right) \geq \mu-\varepsilon
$$

Iterate (2.4) return:

$$
\frac{x\left(g^{k+i}(t)\right)}{x(g(t))} \geq d_{1}^{-(k+i-1)} \prod_{j=1}^{k+i-1} P\left(g^{j}(t)\right)
$$

substitute into (1.1), we have:

$$
x(g(t)) \geq P(t) x(t)+x(g(t)) d_{1}^{-(k+i-1)} \sum_{i=1}^{m} Q_{i}(t) \prod_{j=k+1}^{k+i-1} P\left(g^{j}(t)\right)
$$

By (2.7), we have:

$$
x(g(t)) \geq P(t) x(t)+x(g(t))(\mu-\varepsilon) d_{1}^{-(k+i-1)}
$$

and

$$
\frac{P(t) x(t)}{x(g(t))} \leq 1-\frac{\mu-\varepsilon}{d_{1}^{k+i-1}}=d_{2} .
$$

Substitute $d_{2}, \cdots, d_{n}$ into (2.4) and iterate in turn, we have:

$$
\frac{P(t) x(t)}{x(g(t))} \leq 1-\frac{\mu-\varepsilon}{d_{n-1}^{k+i-1}}=d_{n}
$$

By this we can know that it always has:

$$
\omega_{1}(t)=\frac{P(t) x(t)}{x(g(t))} \leq \frac{d_{n}^{-(k+i-1)}-(\mu-\varepsilon)}{d_{n}^{-(k+i-1)}} . .
$$

We can see that is a decreasing function of the item by item by above, so it's $\lim _{n \rightarrow \infty} d_{n}=d$.

By $\lim _{n \rightarrow \infty} d_{n}=d$ and $1-\frac{\mu-\varepsilon}{d_{n-1}^{k+i-1}}=d_{n}$ we have: When $d_{n}, d_{n-1} \rightarrow d$ multiply $d^{k+i-1}$ on both sides of the equation such that $d, \mu$ satisfied $d^{k+i}-d^{k+i-1}+(\mu-\varepsilon)=0$. Then we have:

$$
\mu-\varepsilon=d^{k+i-1}(1-d)
$$

Substitute (2.10) into (2.9), the proof of (2.3) is completed.

Next, proof $i=2$ by (1.1). We have:

$$
\begin{gathered}
x(g(t)) \geq \sum_{i=1}^{m} Q_{i}(t) x\left(g^{k+i}(t)\right) \\
\omega_{2}(t)=\frac{x\left(g^{2}(t)\right)}{x(g(t))} \sum_{i=1}^{m} Q_{i}(t) \prod_{j=2}^{k+i-1} P\left(g^{j}(t)\right) \leq 1=d_{1} \\
\frac{x\left(g^{k+i}(t)\right)}{x\left(g^{2}(t)\right)} \geq d_{1}^{-(k+i-2)} \prod_{j=2}^{k+i-1} P\left(g^{j}(t)\right)
\end{gathered}
$$


Substituting (1.1), we obtain:

$$
\begin{gathered}
x\left(g^{2}(t)\right) \geq P(g(t)) x(g(t))+x\left(g^{3}(t)\right) \sum_{i=1}^{m} Q_{i}(g(t)) \prod_{j=3}^{k+i-1} P\left(g^{j}(t)\right) \\
1 \geq \frac{P(g(t)) x(g(t))}{x\left(g^{2}(t)\right)}+\frac{x\left(g^{3}(t)\right)}{x\left(g^{2}(t)\right)} \sum_{i=1}^{m} Q_{i}(g(t)) \prod_{j=3}^{k+i-1} P\left(g^{j}(t)\right)
\end{gathered}
$$

and

$$
d_{1}^{-1} \sum_{i=1}^{m} Q_{i}(t) \prod_{j=2}^{k+i-1} P\left(g^{j}(t)\right) \leq \frac{x(g(t))}{x\left(g^{2}(t)\right)}
$$

by

$$
\frac{x\left(g^{2}(t)\right)}{x(g(t))} \sum_{i=1}^{m} Q_{i}(t) \prod_{j=2}^{k+i-1} P\left(g^{j}(t)\right) \leq 1
$$

Hence

$$
1 \geq \frac{\mu-\varepsilon}{d_{1}^{k+i-1}}+\frac{x\left(g^{3}(t)\right)}{x\left(g^{2}(t)\right)} \sum_{i=1}^{m} Q_{i}(g(t)) \prod_{j=3}^{k+i-1} P\left(g^{j}(t)\right)
$$

By

$\omega_{2}(t) \leq \frac{d_{1}^{k+i-1}-(\mu-\varepsilon)}{d_{1}^{k+i-1}}=d_{2}$, calculating in turn we obtain: $\omega_{2}(t) \leq \frac{d_{n}{ }^{k+i-1}-(\mu-\varepsilon)}{d_{n}^{k+i-1}}$.

Equations above show that: $d_{n}$ is a decreasing function of the item by item. Thus $\lim _{n \rightarrow \infty} d_{n}=d$. By $\lim _{n \rightarrow \infty} d_{n}=d$ and $1-\frac{\mu-\varepsilon}{d_{n-1}{ }^{k+i-1}}=d_{n}$ we obtain: When $d_{n}, d_{n-1} \rightarrow d$, we multiply $d^{k+i-1}$ on both sides of the equation, such that $d, \mu$ satisfied $d^{k+i}-d^{k+i-1}+(\mu-\varepsilon)=0$.Substituting (2.10) into $\omega_{2}(t) \leq \frac{d_{n}^{k+i-1}-(\mu-\varepsilon)}{d_{n}^{k+i-1}}$,

We obtain $\limsup _{t \rightarrow \infty} \omega_{2}(t) \leq d$.

Thus, when $0 \leq \mu \leq \frac{k^{k}}{(k+1)^{k+1}}$ and $\varepsilon \rightarrow 0$, equation (1.1) with $x(t)$ is the final positive solutions of equations. Have $\limsup _{t \rightarrow \infty} \omega_{i}(t) \leq d, i=1,2$.

The proof of lemma (1.1) is completed.

Theorem 1.1. When $0 \leq \mu \leq \frac{k^{k}}{(k+1)^{k+1}}$, satisfy

$$
\limsup _{t \rightarrow \infty} \sum_{i=1}^{m} Q_{i}(t) \prod_{j=1}^{k+i-1} P\left(g^{j}(t)\right)>d^{2},
$$

then all solutions of equation (1.1) are oscillatory.

PROOF By (2.9), we obtain:

$$
\limsup _{t \rightarrow \infty} \frac{P(g(t)) x(g(t))}{x\left(g^{2}(t)\right)} \leq d
$$

and

$$
\limsup _{t \rightarrow \infty} \frac{x\left(g^{2}(t)\right)}{x(g(t))} \sum_{i=1}^{m} Q_{i}(t) \prod_{j=2}^{k+i-1} P\left(g^{2}(t)\right) \leq d,
$$

Multiply $\frac{p(g(t)) x(g(t))}{x\left(g^{2}(t)\right)}$ by $\frac{x\left(g^{2}(t)\right)}{x(g(t))} \sum_{i=1}^{m} Q_{i}(t) \prod_{j=2}^{k+i-1} P\left(g^{2}(t)\right) \quad$ will certainly exist 


$$
\sum_{i=1}^{m} Q_{i}(t) \prod_{j=1}^{k+i-1} P\left(g^{2}(t)\right) \leq d^{2}
$$

By Lemma (1.1) we know that $x(t)$ is the eventually positive solution of equation (1.1) when it is in $0 \leq \mu \leq \frac{k^{k}}{(k+1)^{k+1}}$ and $\sum_{i=1}^{m} Q_{i}(t) \prod_{j=1}^{k+i-1} P\left(g^{2}(t)\right) \leq d^{2}$.

By this we have:

When $\quad k \geq 1, m \geq 1 \quad, \quad 0 \leq \mu \leq \frac{k^{k}}{(k+1)^{k+1}} \quad$, every $\quad$ solution $\quad$ of equation $\quad(1.1)$ is oscillatory in $\limsup _{t \rightarrow \infty} \sum_{i=1}^{m} Q_{i}(t) \prod_{j=1}^{k+i-1} P\left(g^{j}(t)\right)>d^{2}$.The proof of Theorem (1.1) is completed.

Theorem 1.2 In equation(1.1), when $0 \leq \mu \leq \frac{k^{k}}{(k+1)^{k+1}}$, the integer $k \geq 0$, it satisfied:

$\limsup _{t \rightarrow \infty}\left\{\bar{\mu}_{\varepsilon}^{k+i-1} \sum_{i=1}^{m} Q_{i}(t) \prod_{j=1}^{k+i-1} P\left(g^{j}(t)\right)+\bar{\mu}_{\varepsilon}^{k+i-1} \sum_{i=1}^{m} Q_{i}(g(t)) \prod_{j=2}^{k+i} P\left(g^{j}(t)\right)\right\}>1$

Then, when $\bar{\mu}=d^{-1}$ all of the solutions of equation (1.1) are oscillatory.

PROOF: Assume that (1.1) has a solution. In lemma 1.1, there is an $\varepsilon>0$, and $t$ Is as large as possible, such that:

$$
x(g(t)) \geq \overline{\mu_{\varepsilon}} P(t) x(t)
$$

By (2.11) iteration, we get:

$$
\begin{gathered}
x\left(g^{k+i}(t)\right) \geq\left(\bar{\mu}_{\varepsilon}^{k+i-1} \prod_{j=1}^{k+i-1} P\left(g^{j}(t)\right) x(g(t))\right. \\
x(g(t)) \geq \overline{\mu_{\varepsilon}} \sum_{i=1}^{m} Q_{i}(t) x\left(g^{k+i}(t)\right)
\end{gathered}
$$

Have: $x\left(g^{2}(t)\right)=P(g(t)) x(g(t))+\sum_{i=1}^{m} Q_{i}(g(t)) x\left(g^{k+i+1}(t)\right)$

Obtain: $x\left(g^{2}(t)\right) \geq P(g(t)) \overline{\mu_{\varepsilon}} \sum_{i=1}^{m} Q_{i}(t) x\left(g^{k+i}(t)\right)+\sum_{i=1}^{m} Q_{i}(g(t)) x\left(g^{k+i+1}(t)\right)$

$x\left(g^{2}(t)\right) \geq x\left(g^{2}(t)\right) \bar{\mu}_{\varepsilon}^{k+i-1} \sum_{i=1}^{m} Q_{i}(t) \prod_{j=1}^{k+i-1} P\left(g^{j}(t)\right)+x\left(g^{2}(t)\right) \bar{\mu}_{\varepsilon}^{k+i-1} \sum_{i=1}^{m} Q_{i}(g(t)) \prod_{j=2}^{k+i} P\left(g^{j}(t)\right)$

Finally obtain:

$$
1 \geq \bar{\mu}_{\varepsilon}^{k+i-1} \sum_{i=1}^{m} Q_{i}(t) \prod_{j=1}^{k+i-1} P\left(g^{j}(t)\right)+\bar{\mu}_{\varepsilon}^{k+i-1} \sum_{i=1}^{m} Q_{i}(g(t)) \prod_{j=2}^{k+i} P\left(g^{j}(t)\right) .
$$

When $t \rightarrow \infty$, we can obtain that equation (1.1) have finally positive solution when

$$
\limsup _{t \rightarrow \infty}\left\{\bar{\mu}_{\varepsilon}^{k+i-1} \sum_{i=1}^{m} Q_{i}(t) \prod_{j=1}^{k+i-1} P\left(g^{j}(t)\right)+\bar{\mu}_{\varepsilon}^{k+i-1} \sum_{i=1}^{m} Q_{i}(g(t)) \prod_{j=2}^{k+i} P\left(g^{j}(t)\right)\right\} \leq 1
$$

The proof is completed. 


\section{Examples}

$$
x(t+\pi)=\frac{1}{t} x(t)+\frac{t+\pi}{100} x(t+2 \pi)+t^{2}\left(\frac{1}{8}+\frac{3}{5} \cos ^{2} t\right) x(t+3 \pi), g=t+\pi
$$

PROOF: By the question, we know

$$
\begin{gathered}
P(t) \equiv \frac{1}{t}, Q_{1}(t)=\frac{t+\pi}{100}, Q_{2}(t)=t^{2}\left(\frac{1}{8}+\frac{3}{5} \cos ^{2} t\right), \\
\inf \sum_{i=1}^{2} \mathrm{Q}_{\mathrm{i}}(t) \prod_{j=1}^{2} P\left(g^{j}(t)\right)=\left(\frac{t+\pi}{100}+t^{2} \frac{1}{8}\right) \frac{1}{t^{2}}=\frac{t+\pi}{100 t^{2}}+\frac{1}{8} .
\end{gathered}
$$

Then

$$
\begin{gathered}
\lim _{t \rightarrow \infty} \inf \sum_{i=1}^{2} \mathrm{Q}_{\mathrm{i}}(t) \prod_{j=1}^{2} P\left(g^{j}(t)\right)=\frac{1}{8}<\frac{1}{4} . \\
\sup \sum_{i=1}^{2} \mathrm{Q}_{\mathrm{i}}(t) \prod_{j=1}^{2} P\left(g^{j}(t)\right)=\left[\frac{t+\pi}{100}+t^{2}\left(\frac{1}{8}+\frac{3}{5}\right)\right] \frac{1}{t^{2}}=\frac{t+\pi}{100 t^{2}}+\frac{29}{40} .
\end{gathered}
$$

Then

$$
\lim _{\mathrm{t} \rightarrow \infty} \sup \sum_{i=1}^{2} \mathrm{Q}_{\mathrm{i}}(t) \prod_{j=1}^{2} P\left(g^{j}(t)\right)=\frac{29}{40}<1 .
$$

Let

$$
\mu=\liminf _{t \rightarrow \infty} \sum_{i=1}^{m} Q_{i}(t) \prod_{j=1}^{k+i-1} P\left(g^{j}(t)\right)
$$

As well as because when $d^{k+i}-d^{k+i-1}+\mu=0, k=1, i=2, \quad d^{3}-d^{2}+\frac{1}{8}=0$, we could obtain $d=\frac{1}{2}$.

By $\limsup _{t \rightarrow \infty} \sum_{i=1}^{2} Q_{i}(t) \prod_{j=1}^{2} P\left(g^{j}(t)\right)>d^{2}$ obtain that

$$
\limsup _{t \rightarrow \infty} \sum_{i=1}^{2} Q_{i}(t) \prod_{j=1}^{2} P\left(g^{j}(t)\right) \rightarrow \frac{29}{40}>\frac{1}{4} .
$$

It can prove that the solution of equation (3.1) is oscillatory for a large $t$.

\section{Acknowledgments}

This research is supported by Science of School, Guangdong University of Petrochemical Technology (2018001) and Maoming City Science and Technology Plan Project of Guangdong Province (2015038) P.R. China. We also want to thank the commentators for their constructive comments on the paper.

\section{References}

DAI, L. N., WU, Y. Z., \& LIN, Q. W. (2014). Oscillatory Behavior of Solutions to Higher Order Variable Coefficient Functional Equations. Mathematics in Practice and Theory, 44(10), 271-275.

Golda, W., \& Werbowski, J. (1994). Oscillation of linear functional equations of the second order. Funkcialaj Ekvacioj, 37(2), 221-227.

Jinghua, S., \& Stavroulakis, I. P. (2002). An oscillation criteria for second order functional equations. Acta Mathematica Scientia, 22(1), 56-62. https://doi.org/10.1016/S0252-9602(17)30455-1

Lin, Q. W., WU, Y. Z., \& Liao, S. Q. (2009). The oscillation of nonlinear functional equations with variable coefficients. Math. Research, 2, 216-221. https://doi.org/10.5539/jmr.v1n2p216

Lou, Z. R., \& Shen, J. H. (2003). New results for oscillation of Higher order linear functional equations. Journal of Systerms Science \& Mathematical Science, 23(4), 508-516.

Nowkowska, W., \& Werbowski, J. (1995). Oscillation of functional equations of higher order. Arch Math, 31, 251-258.

Nowkowska, W., \& Werbowski, J. (2001). Osciillation behavior of solutions of functional equations. Nonlinear Analysis, 44, 767-775. https://doi.org/10.1016/S0362-546X(99)00306-5

Si-Min, W. U., Dai, L. N., Lin, Q. W., Mathematics, D. O., \& Science, S. O. (2013). Nonoscillatory criteria of solutions 
to higher order functional equations. Mathematics in Practice \& Theory, 43(20), 280-285.

Yong, Z., \& Yuanhong, Y. (1999). Oscillation of functional equations with variable coefficients. System Science and Mathematics, 19(3), 348-352.

Zhang, B. G., \& Choi, S. K. (2001). An oscillation and non-oscillation of a class of functional equations.Mathematische Nachrichten, (227), 159-169. https://doi.org/10.1002/1522-2616(200107)227:1<159::AID-MANA159>3.0.CO;2-D

Zhang, X. L., \& Lou, Z. R. (2003). Oscillatory behavior of higher order linear functional equations. Acta Mathematicae Applicatae Sinica, 26(1), 186-189.

Zhou, Y., Liu, Z. R., \& Yu, Y. H. (2000). Oscillation criteria of functional equations with variable coefficients. Journal of Dongguan University of Technology, (3), 413-419.

\section{Copyrights}

Copyright for this article is retained by the author(s), with first publication rights granted to the journal.

This is an open-access article distributed under the terms and conditions of the Creative Commons Attribution license (http://creativecommons.org/licenses/by/4.0/). 